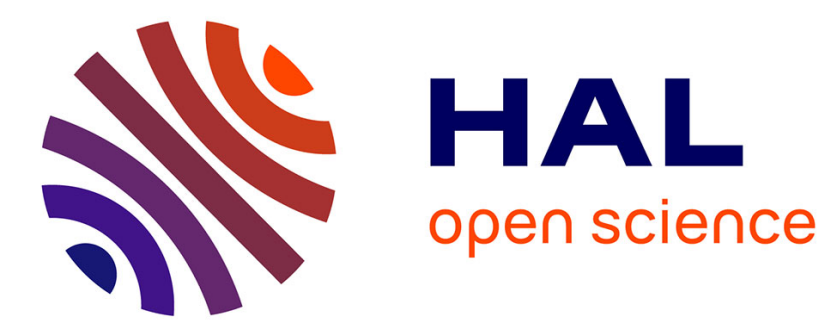

\title{
Outage Analysis of Integrated Mesh LTE Femtocell Networks
}

\author{
Anthony Busson, Lynda Zitoune, Véronique Vèque, Bijan Jabbari
}

\section{To cite this version:}

Anthony Busson, Lynda Zitoune, Véronique Vèque, Bijan Jabbari. Outage Analysis of Integrated Mesh LTE Femtocell Networks. IEEE Global Communications Conference GLOBECOM 2014, Dec 2014, Austin, United States. pp.1-6, 10.1109/glocom.2014.7036805 . hal-00979627v1

\section{HAL Id: hal-00979627 \\ https://hal-centralesupelec.archives-ouvertes.fr/hal-00979627v1}

Submitted on 16 Apr 2014 (v1), last revised 26 Oct 2018 (v2)

HAL is a multi-disciplinary open access archive for the deposit and dissemination of scientific research documents, whether they are published or not. The documents may come from teaching and research institutions in France or abroad, or from public or private research centers.
L'archive ouverte pluridisciplinaire HAL, est destinée au dépôt et à la diffusion de documents scientifiques de niveau recherche, publiés ou non, émanant des établissements d'enseignement et de recherche français ou étrangers, des laboratoires publics ou privés. 


\title{
Outage Analysis of Integrated Mesh LTE Femtocell Networks
}

\author{
Anthony Busson*, Lynda Zitoune ${ }^{\dagger}$, Véronique Vèque ${ }^{\ddagger}$ and Bijan Jabbari ${ }^{\S}$ \\ *Laboratoire de l'Informatique du Parallèlisme \\ 46 Allée d'Italie, Lyon 69364, France. Email: Anthony.Busson@ens-lyon.fr \\ ${ }^{\dagger}$ Department of Systems Engineering, ESIEE-Paris \\ 2, boulevard Blaise Pascal, 93162 Noisy le Grand, France. Email: lynda.zitoune@esiee.fr \\ ${ }^{\ddagger}$ Signals and Systems Laboratory, SUPELEC \\ 3, Rue Joliot-Curie, 91192 Gif sur Yvette, France. Email: Veronique.VEQUE@1ss.supelec.fr \\ $\S$ Electrical and Computer Engineering Department \\ George Mason University Fairfax, VA 22030, USA. Email: bjabbari@gmu.edu
}

\begin{abstract}
Using Wi-Fi with femtocell is a promising approach to solve the problem of inter-tier or intra-tier interference in heterogeneous networks. In our scenario, Wi-Fi femtocells are deployed to form a mesh network (Wi-Fi) which is used by mobile users as an access network to the macro cellular one (e.g., LTE), extending consequently, the coverage to poorly covered areas. We propose a tractable model for coverage/outage to evaluate the benefits of such integration in terms of SINR and received signal strength. Our work is based on point processes in two-dimensional plane that models locations of femtocell/WiFi nodes (also referred to as multimode nodes). The proposed model is more realistic than the classical Poisson point process, as the distribution of points is more homogeneous and it ensures that the nodes are not too close to each other. The derivation of coverage/outage formula allows us to determine operational parameter ranges for the Wi-Fi network to form a mesh network. In addition, it helps in the design of the femtocell network to ensure a suitable coverage for users in terms of SINR and received signal strength.
\end{abstract}

\section{INTRODUCTION}

Femtocell networks and more generally small cell networks are promising solutions to address the growth of the user's traffic density, diversity and coverage. The subject has received considerable attention in recent years [1]-[5] from both academia and industry. Typically, femtocells (FTCs), operating in the licensed spectrum, are used to reduce the distance between the mobile user and the macro BS to which it belongs, using an efficient spatial reuse spectrum mechanisms [4], [6]. Consequently, indoor users may experience higher quality wireless services, and outdoor users can gain access to higher capacity.

Femtocells used with macrocells together form a heterogeneous network (also called k-tier network) to improve the network performance in terms of coverage and capacity. However, in practice communication suffers from the interference induced by FTCs when used in 1-tier network alone or in the 2-tier case. The interference is due to the spectrum sharing and the random location of the FTCs being user dependent. Several techniques have recently been deployed to handle the interference problem between the macro and small cells including power control, multiple antennas, adaptive FAP
(Femtocell Access Point) access scheme, cognitive radio and spectrum allocation [3].

Other technical and economic/regulatory challenges are still present within femtocell networks when applied in outdoors, these include cell association and biasing, mobility and soft handoff, self-organizing (see [4] for more details). In [7], the authors state that to take advantage of femtocells, innovative solutions must be developed to deal with the challenges cited above, and propose to integrate multiple radio access technologies (RAT), for example the integration of cellular (LTE) and Wi-Fi technologies. Thus, it is not surprising that the next generation of small cell BS is expected to be multi-mode. Consequently, the femtocell-Wi-Fi integration may resolve the problem of inter-tier or intra-tier interference by offloading some of the traffic to the unlicensed and shared-band of WiFi. Conversely, when contention increases causing throughput reduction on the shared Wi-Fi band, some of the traffic can be switched to cellular links. This integration may provide benefits resulting from these two technologies, as summarized in [8].

In this paper, we aim to study coverage and outage of a network of nodes which integrate both femtocell and WiFi RAT. We consider a particular scenario as depicted in Figure I. Only one macro base station is deployed, and we aim to extend the coverage of this macro to poorly covered (or uncovered) areas. It's typically the case of rural region which has no real communication infrastructure such as ADSL. The solution we consider, is to deploy some multimode FTCs, such that the so formed mesh Wi-Fi network can be used as an access network for the users which are very far from the macro base station. We assume that a multimode FTC implement both cellular (e.g., LTE), and Wi-Fi technologies. Communications of mobile users are carried through using FTC to which they belong. The Wi-Fi mesh network is used to ensure the connectivity between mobile users and their macro BS.

We propose an innovative spatial point process to model this network. It differs from the classical Poisson Point Process and other work on femtocells [1]-[3], [5] because points are 


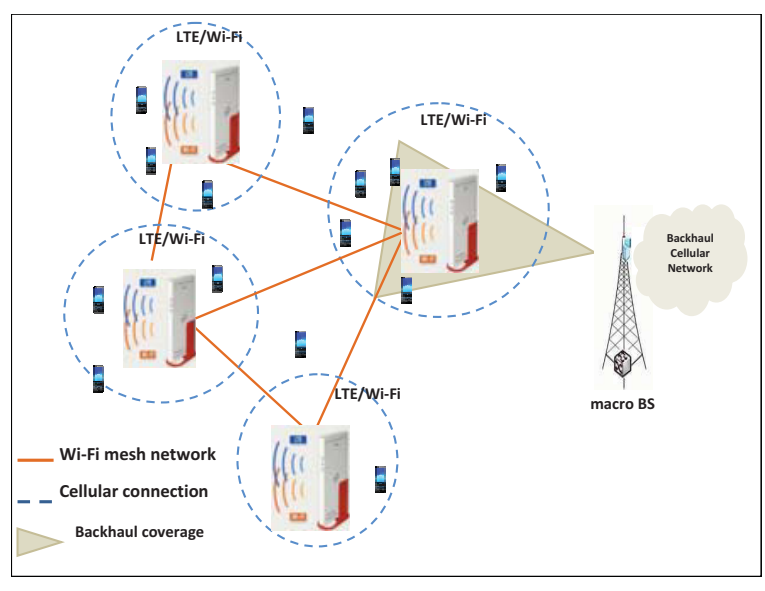

Fig. 1. Outdoor coverage network in poorly covered areas.

more regularly distributed in the plane leading to a more uniform coverage. Furthermore, we prevent the presence of FTCs which are too close to each other. The present work may be applied in two contexts: 1) a dimensioning tool to evaluate ideal/optimal outdoor deployment of multimode FTCs and 2) in green-networking where certain FTCs have to be switchedoff to save energy. For the latter, our model offers analytical capability to evaluate location and number of multimode FTCs that are needed to be kept on, while ensuring an accurate coverage of the cellular network and the connectivity over the mesh Wi-Fi network.

\section{A. Related work}

Stochastic geometry is extensively used to develop more tractable analytical models to study the performance of heterogeneous or k-tier cellular networks, in terms of the probability of coverage, outage and throughput. Practically, most of modeling papers consider Poisson Point Process (PPP) to describe the position of the FTCs and the mobile users.

In [1], the authors developed a general expression of the coverage probability in a 1-tier cellular network where the interference fading/shadowing follows an arbitrary distribution. They study the coverage through the signal on interference plus noise ratio (SINR), a region being covered if a typical user experiences a SINR greater than a threshold with a certain probability. They consider Rayleigh fading, and ignore the thermal noise. The authors derive the mean achievable rate and study coverage and rate in order to find the required frequency reuse to reach a specified coverage probability.

The paper [9] extends the model of coverage probability of [1] to consider multi-tier cellular network, by modeling downlink SINR. This new model has been compared to an actual 4G macro-cell network to validate its accuracy.

In [2], the same authors evaluate the impact of different cell association policies on metrics like the minimum average user throughput and the average ergodic rate. They assume that a mobile user connects to the FTC that offers the best long-term averaged received power rather than the greatest instantaneous SINR. In this work, the outage probability is also calculated, and they observe that neither the number of BS nor tiers change the outage probability and the ergodic rate in an interference-limited fully-loaded heterogeneous cellular network with unbiased cell association. In [5], the authors argue that interference is more important in the "inner regions" close to the macro BS. So, they propose to deactivate FTCs in zones where there is sufficient macrocell coverage. They show the benefits of their solution on the downlink coverage.

The authors in [5] define an analytical model which describes the coverage probability at a randomly located mobile user. In the first step, the model (same as [9]) describes the coverage probability for uniform femtocell deployment, where all the FTCs remain active.Then, they derive another model for the overall coverage probability by defining the coverage probability in both the inner (where FTCs are inactive) and outer regions (where FTCs are active). This new model depends on the radius of the inner region. Monte Carlo simulations are also conducted to validate the model.

The model of [1] is also used in [3] to handle the problem of inter-tier interference between a macrocell BS and FTCs in case of severe frequency reuse. The aim is to assess the impact of spectrum allocation and the femtocell access policies on the link reliability of each tier and on the total network throughput.

\section{B. Approach and Contributions}

We consider an outdoor deployment of multimode FTCs to extend the coverage of one macro base station in rural region. A set of multimode (LTE/Wi-Fi) FTCs distributed arbitrarily over the plane. Our aim is to switch off some of them in order to save energy and at the same time to reduce interference on mobile users at the edge of the cell. The switching off process must guarantee the Wi-Fi connectivity between the remaining FTCs. As a result, we form a Wi-Fi mesh network of FTCs that serves as an access network of the mobile nodes which are distant from the macro base station.

The point process we propose aims to model locations of the FTCs that are active. In order to ensure an accurate coverage, FTCs cannot be switched off randomly and independently of each other, because it could lead to uncovered regions or regions with FTCs interfering with each other. Consequently, the Poisson Point Process is inaccurate in this context. Furthermore, locations of the FTCs being known, since they are deployed local organizations like city hall, or schools, it is possible to keep a set of FTCs that covers the plane/region more or less regularly. Therefore, we split the plane into a set of squares of same size, forming a partition. For each square, we keep only one FTC on to ensure a uniform coverage of the plane. But, two FTCs in two adjacent squares may still be arbitrarily close to each other generating redundant coverage and harmful interference. As the choice of active $s$ may be planned in advance, and with the knowledge of the FTCs location, this kind of situations should not appear. The idea is then to consider that these FTCs are distributed in the core of each square and not on the edges. This type of point process belongs to the family of the so-called hard-core point process. With these point processes, the distance between two points 


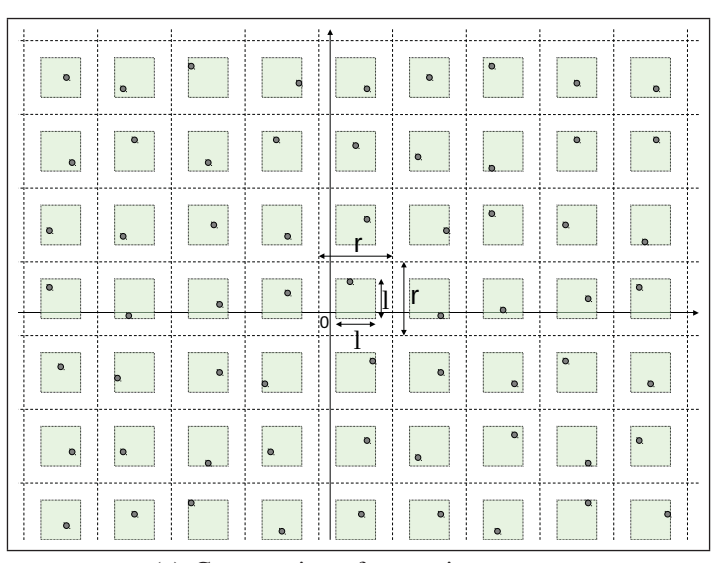

(a) Construction of our point process

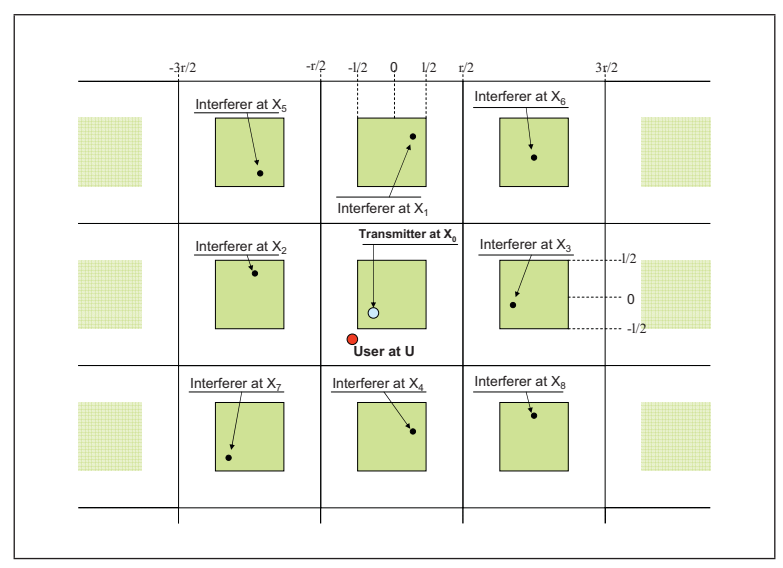

(b) Interferents and transmitter locations/numbering taken into accoun in the SINR computation

Fig. 2. Notations

cannot be less than a given threshold. The most used hardcore point processes in the Telecommunication domain are the Simple Sequential Inhibition [10], or Matèrn [11], but for which analytical derivations of Interference or SINR distribution are intractable. The point process that we propose has the benefit that it models realistically the FTC selection (saving energy while ensuring a good coverage), and is mathematically tractable.

The remainder of the paper is organized as follows. In section II, we present the point process used to model the integrated FTC/Wi-Fi network. In section III, we provide tractable analytical formulas of SINR and received signal strength at a mobile user. We present the numerical results in Section IV. Finally concluding remarks are made in Section V.

\section{FTC/Wi-Fi Coverage Model}

The proposed point process may model both the FTC and Wi-Fi networks. Path-loss and transmission power levels can be adapted with respect to the considered technology. In the following, we shall use the term node or point rather than FTC or Access Point, as the nodes implement these two technologies. The point process represents the node locations for nodes that have not been switched-off. We consider a partition of the plane formed by a grid of size $r \times r$. In order to keep the process stationary, the grid is not centered at 0 , but is centered at a point randomly distributed in the interval $[0, r] \times[0, r]$. In each of these squares, we set a sub-square of size $l \times l$ (with $0 \leq l \leq r$ ) at the center. A point is then uniformly distributed in each sub-square. When $l<r$, this process is a hard core point process, as the points cannot lie at a distance less than $r-l$. In our context, it guarantees that points in two adjacent squares cannot be too close to each other. It limits interference between adjacent cells. Figure 2(a) illustrates the construction of this process and the different notations. The intensity of this point process is then $\frac{1}{r}$ whatever the value of $l$.

Mobile users are set according to a Poisson point process distributed in the plane. Conditioned on the presence of a user in a square, this mobile user is thus uniformly distributed within this square. Without loss of generality we assume that this point is located at the origin (by construction the origin is uniformly distributed in the square covering it). Also, we assume that a mobile user associates and communicates with the closest FTC. Each FTC is thus responsible for users distributed in its Voronoï cell. These Voronoï cells are more or less regular. It depends on the ratio $\frac{l}{r}$ between small and big squares. The impact of this ratio may be observed in Figure II, where we plotted the Voronoï cells of this point process for different $\frac{l}{r}$ ratios.

We focus on the downlink between the FTC and the mobile user. Interference is then computed at the user location. Interference at a FTC can be derived in the same way, but is not considered in this paper. Interference will be mainly generated by the FTC within the adjacent squares of the mobile user. Consequently, we estimate interference only from these squares. For convenience, we shift the center of the square where the mobile user is located at the origin. As shown in Figure 2(b), the squares are numbered from 0 (square at the origin) to 8 . Locations of the associated point are denoted $X_{0}, X_{1}, \ldots, X_{8}$. The corresponding $r \times r$ and $l \times l$ squares are denoted $R_{0}, R_{1}, . ., R_{8}$, and $L_{0}, L_{1}, . ., L_{8}$ respectively. The mobile user location is denoted with the random variable $U$. It follows a uniform distribution in the square $0\left(\left[-\frac{r}{2}, \frac{r}{2}\right] \times\left[-\frac{r}{2}, \frac{r}{2}\right]\right)$. In our example, the closest FTC from the user is the point in the square 0. For this example, the transmission takes place between the point at $U$ and $X_{0}$, and interference are generated by points at $X_{1}, . ., X_{8}$.

Let $K(K \in\{0, . ., 8\})$ be the discrete random variable equal to the index of the closest femtocell/points from $U$, and $I(U)$ the interference at the user, we get:

$$
I(U)=\sum_{i=0}^{8} P_{t} \xi_{i} l\left(\left\|U-X_{i}\right\|\right) \mathbb{1}_{K \neq i}
$$

where $P_{t}$ is the transmission power (it is assumed to be the same for all femtocells), $\left(\xi_{i}\right)_{i}$ is a sequence of i.i.d. random 


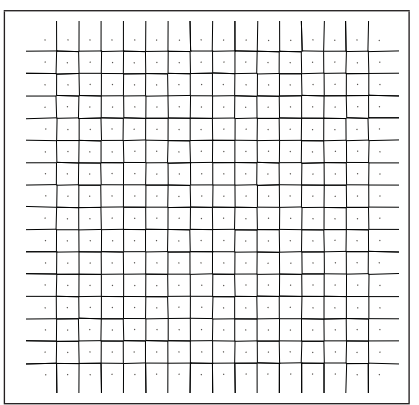

(a) $\frac{l}{r}=0.033$

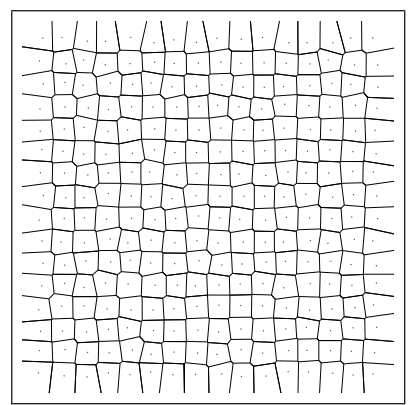

(b) $\frac{l}{r}=0.25$

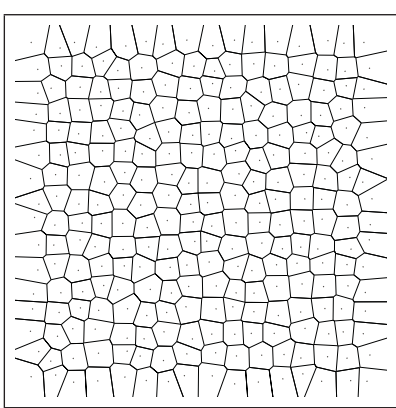

(c) $\frac{l}{r}=0.5$

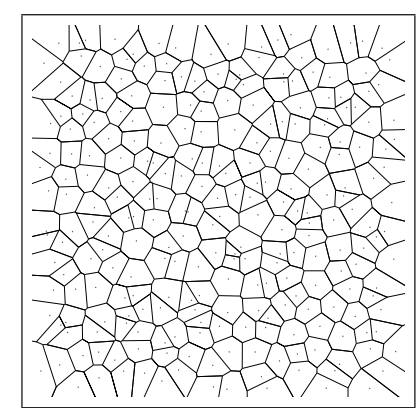

(d) $\frac{l}{r}=1.0$

Fig. 3. Impact of the ratio $\frac{l}{r}$ on the Voronoï cells.

variables modeling fading, $l($.$) is the path-loss function and$ $\mathbb{1}$. is the indicator function. The Signal over Interference plus Noise Ratio (SINR), is then given by:

$$
S I N R=\frac{\xi_{K} l\left(\left\|X_{K}-U\right\|\right)}{N+I(U)}
$$

where $N$ is a random variable modeling the noise. In the following, we consider a Rayleigh fading where $\left(\xi_{i}\right)_{i}$ are independently and exponentially distributed with parameter 1 .

\section{iII. Performance Evaluation}

\section{A. Coverage/Outage}

We consider the distribution of two main quantities that impact the design of the integrated FTC/Wi-Fi network: the SINR and the received signal strength. The proof is given only for the SINR distribution. The other computations are very similar.

Proposition 1. The complementary cumulative distribution function $(C C D F)$ of the SINR is given by:

$$
\begin{aligned}
& \mathbb{P}(\operatorname{SINR}>\beta) \\
& =\frac{1}{r^{2} l^{2}} \int_{R_{0}} \sum_{k=0,1,5} C_{k} \int_{L_{k}} \mathbb{E}\left[e^{\left.-\frac{\beta N}{P_{t} l\left(\left\|x_{k}-u\right\|\right)}\right]}\right. \\
& \times \prod_{i=0 ; i \neq k}^{8} \mathbb{E}\left[\frac{\mathbb{1}_{X_{i} \notin B\left(u,\left\|x_{k}-u\right\|\right)}}{1+\beta \frac{l\left(\left\|X_{i}-u\right\|\right)}{l\left(\left\|x_{k}-u\right\|\right)}}\right] d x_{k} d u
\end{aligned}
$$

with $C_{0}=1$, and $C_{1}=C_{5}=4$. The CCDF of the received signal strength is given by:

$$
\begin{aligned}
\mathbb{P} & \left(\xi_{K} P_{t} l\left(\left\|X_{k}-U\right\|\right)>\beta\right) \\
& =\frac{1}{r^{2} l^{2}} \int_{R_{0}} \sum_{k=0,1,5} C_{k} \int_{L_{k}} e^{-\frac{\beta}{P_{t} l\left(\left\|x_{k}-u\right\|\right)}} \\
& \times \prod_{i=0 ; i \neq k}^{8} \mathbb{P}\left(X_{i} \notin B\left(u,\left\|x_{k}-u\right\|\right)\right) d x_{k} d u
\end{aligned}
$$

or when we consider the signal strength without fading:

$$
\begin{aligned}
\mathbb{P} & \left(P_{t} l\left(\left\|X_{k}-U\right\|\right)>\beta\right) \\
& =\frac{1}{r^{2} l^{2}} \int_{R_{0}} \sum_{k=0,1,5} C_{k} \int_{L_{k}} \mathbb{1}_{P_{t} l\left(\left\|x_{k}-u\right\|\right)>\beta} \\
& \times \prod_{i=0 ; i \neq k}^{8} \mathbb{P}\left(X_{i} \notin B\left(u,\left\|x_{k}-u\right\|\right)\right) d x_{k} d u
\end{aligned}
$$

Proof:

$$
\begin{aligned}
\mathbb{P} & (S I N R>\beta)=\mathbb{P}\left(\frac{P_{t} l\left(\left\|X_{K}-U\right\|\right) \xi}{N+I}>\beta\right) \\
& =\mathbb{P}\left(\xi>\frac{\beta(N+I)}{P_{t} l\left(\left\|X_{K}-U\right\|\right)}\right) \\
& =\mathbb{E}\left[e^{-\frac{\beta(N+I)}{P_{t} l\left(\left\|X_{K}-U\right\|\right)}}\right] \\
& =\sum_{k=0}^{k=8} \mathbb{E}\left[e^{-\frac{\beta(N+I)}{P_{t} l\left(\left\|X_{i}-U\right\|\right)}} \mathbb{1}_{K=i}\right]
\end{aligned}
$$

By simple symmetric arguments, we can see that the terms in the sum of Equation (11) are equal for all possible values of $K$ in $\{1,2,3,4\}$ and $\{5,6,7,8\}$. Therefore, we can distinguish three different cases: $K=0, K=1$, and $K=5$. If we set the constant $C_{0}=1$ and $C_{1}=C_{5}=4$, we get,

$$
\begin{aligned}
& \mathbb{P}(S I N R>\beta) \\
& \quad=\sum_{k=0,1,5} C_{i} \mathbb{E}\left[e^{-\frac{\beta(N+I)}{P_{t} l\left(\left\|X_{k}-U\right\|\right)}} \mathbb{1}_{K=k}\right]
\end{aligned}
$$

We condition by $U$, and each term of the sum by the transmitter location, $\left(X_{0}, X_{1}\right.$ or $\left.X_{5}\right)$ :

$$
\begin{aligned}
& \mathbb{P}(S I N R>\beta) \\
& =\frac{1}{\left|R_{0}\right|} \frac{1}{\left|L_{0}\right|} \int_{R_{0}} \sum_{k=0,1,5} C_{k} \int_{L_{k}} \mathbb{E}\left[e^{-\frac{\beta(N+I)}{P_{t}\left(\left\|x_{k}-u\right\|\right)}} \mathbb{1}_{K=k}\right] d x_{k} d u
\end{aligned}
$$

Computation of the expectations can be done as follows. Let $k \in\{0,1,5\}$, we obtain:

$$
\begin{aligned}
& \mathbb{E}\left[e^{-\frac{\beta(N+I)}{P_{t} l\left(\left\|x_{k}-u\right\|\right)}} \mathbb{1}_{K=k}\right] \\
& \quad=\mathbb{E}\left[e^{\left.-\frac{\beta N}{P_{t} l\left(\left\|x_{k}-u\right\|\right)}\right]} \mathbb{E}\left[e^{-\frac{\beta I}{P_{t} l\left(\left\|x_{k}-u\right\|\right)}} \mathbb{1}_{K=k}\right]\right. \\
& \quad=\mathbb{E}\left[e^{-\frac{\beta N}{P_{t} l\left(\left\|x_{k}-u\right\|\right)}}\right] \mathbb{E}\left[e^{-\frac{\beta \sum_{i=0 ; i \neq k}^{8} \xi_{i} P_{t} l\left(\left\|X_{i}-u\right\|\right)}{P_{t} l\left(\left\|x_{k}-u\right\|\right)}} \mathbb{1}_{K=k}\right]
\end{aligned}
$$

The second term is as follows: 


\begin{tabular}{|c|c|}
\hline Parameters & Values \\
\hline \hline Path-loss function $(\mathrm{dBm})$ & $l(d)=\min \left(C, C-10 \alpha \log _{10}(d)\right)$ \\
\hline$\alpha$ & 3.0 \\
\hline$C$ & $3.76 e^{-5}$ \\
\hline$P_{t}$ & $-17 \mathrm{dBm}(50 \mathrm{~mW})$ \\
\hline Receiver sensitivity & Normal $\left(1.0 e^{-11}, 3.76 e^{-11}\right)$ \\
\hline Noise $(\mathrm{mW})$ &
\end{tabular}

TABLE I

PARAMETERS FOR THE NUMERICAL EVALUATION.

$$
\begin{aligned}
\mathbb{E} & {\left[e^{-\frac{\beta \sum_{i=0 ; i \neq k}^{8} \xi_{i} P_{t} l\left(\left\|X_{i}-u\right\|\right)}{P_{t} l\left(\left\|x_{k}-u\right\|\right)}} \mathbb{1}_{K=k}\right] } \\
& =\prod_{i=0 ; i \neq k}^{8} \mathbb{E}\left[e^{-\frac{\beta \xi_{i} l\left(\left\|X_{i}-u\right\|\right)}{l\left(\left\|x_{k}-u\right\|\right)}} \mathbb{1}_{X_{i} \notin B\left(u,\left\|x_{k}-u\right\|\right)}\right] \\
& =\prod_{i=0 ; i \neq k}^{8} \mathbb{E}\left[\frac{\mathbb{1}_{X_{i} \notin B\left(u,\left\|x_{k}-u\right\|\right)}}{1+\beta \frac{l\left(\left\|X_{i}-u\right\|\right)}{l\left(\left\|x_{k}-u\right\|\right)}}\right]
\end{aligned}
$$

Finally, we obtain:

$$
\begin{aligned}
& \mathbb{P}(\operatorname{SINR}>\beta) \\
& =\frac{1}{\left|R_{0}\right|} \frac{1}{\left|L_{0}\right|} \int_{R_{0}} \sum_{k=0,1,5} C_{k} \int_{L_{k}} \mathbb{E}\left[e^{\left.-\frac{\beta N}{P_{t} l\left(\left\|x_{k}-u\right\|\right)}\right]}\right. \\
& \times \prod_{i=0 ; i \neq k}^{8} \mathbb{E}\left[\frac{\mathbb{1}_{X_{i} \notin B\left(u,\left\|x_{k}-u\right\|\right)}}{1+\beta \frac{l\left(\left\|X_{i}-u\right\|\right)}{l\left(\left\|x_{k}-u\right\|\right)}}\right] d x_{k} d u
\end{aligned}
$$

If we assume that the noise follows a Gaussian distribution with mean $\mu$ and variance $\sigma^{2}$, we get:

$$
\begin{aligned}
\mathbb{E} & {\left[e^{\left.-\frac{\beta N}{P_{t} l\left(\left\|x_{k}-u\right\|\right)}\right]}\right.} \\
& =e^{-\frac{1}{2}\left(\frac{\sigma \beta}{P_{t} l\left(\left\|x_{k}-u\right\|\right)}\right)^{2}-\frac{\mu \beta}{P_{t} l\left(\left\|x_{k}-u\right\|\right)}}
\end{aligned}
$$

\section{NUMERICAL EVALUATION}

Our model aims at defining the values of $r$ and $l$, which verify the different constraints of the FTC and Wi-Fi networks. In order to save maximum energy, i.e. to switch-off maximum number of nodes, we need to choose the greatest $r$ that satisfies these constraints. If $\lambda_{f}$ is the initial femtocell intensity, we can easily express the ratio between the remaining femtocell and the number of initial femtocell. as $\frac{1}{\lambda_{f} r^{2}}$. It may give a precise estimate of the saved energy, but this point is not developed in this paper.

The constraints on $r$ are manifold. $r$ must be bounded in order to keep the Wi-Fi network connected. It suffices to guarantee that nodes into two adjacent squares are in the radio range of each others. Therefore, we can choose $r$ to guarantee a maximum distance between two nodes, or ensure that the received signal strength will be greater than a given threshold with a certain probability (as it is developed below for the femtocell network). Interference does not have to be considered here, as the CSMA/CA mechanism used in the Wi-Fi network ensures that two interfering nodes are not transmitting at the same time.

On the other hand, in the femtocell network the medium is shared by nodes lying in adjacent squares. A transmission from a femtocell to a user will be properly received if both the SINR and the received signal strength are sufficiently great. The second criterion is linked to the receiver device sensitivity. For instance, in Figure 4(a) we assumed a fixed receive power target of $-100.0 \mathrm{dBm}$. The other parameters are given in Table I. We have plotted the CCDF of the received signal strength without fading as given by Equation (7) for $\beta=-100$ dBm. $r$ is chosen in such a way that $95 \%$ of the users will experience a signal strength greater than this threshold. This is shown through the horizontal line in the figure. The maximum values of $r$ with respect to this constraint varies from 400 to approximately 570 meters. There is thus an important gain in terms of coverage with respect to the parameter $l$.

Also, we compared these results to the Poisson Point Process with same intensity $\left(\lambda=\frac{1}{r^{2}}\right)$ in the same figure. Due to the fact that distance between the user and its closest node is not bounded for Poisson (the user may be arbitrarily far from the closest node), CCDF of the received signal strength is less for Poisson than for our model. It involves that for a given threshold on this CCDF, the coverage distance is less for Poisson.

The second criterion is the SINR. We need to set $r$ and $l$ in such a way that $P(S I N R>\beta)>1-\epsilon$. In Figure 4(b), we plot the SINR CCDF for $r=400.0$ and different values of $l$ (as given by Equation (3)). We can observe the impact of $l$ on the SINR. Small values of $l$ lead to a significant improvement of SINR. Poisson still leads to a less efficient coverage as SINR is likely to be smaller than for our process.

The SINR CCDF for a given value of $\beta(\mathbb{P}(S I N R>\beta))$ does not vary with $r$. It is not plotted here due to lack of space, and because curves simply consist of horizontal line for both processes. As it has been shown for the Poisson point process [1], when the noise is negligible, this quantity stays constant with respect to $r$ (and $P_{t}$ ) due to the ratio between received signal strength and interference.

Other criteria can be taken into account to set $l$ and $r$ values. Given the initial spatial distribution of the nodes (before they have been switched-off), we may choose $l$ such that we have a reasonable probability to have a femtocell in a square of size $l \times l$. Also, $r$ can be set according to the mean number of users associated with a femtocell. As the femtocell intensity is $\frac{1}{r^{2}}$, the mean size of a Voronoï cell is $r^{2}$. If the users intensity is $\lambda_{u}$, then the mean number of associated users is $\lambda_{u} r^{2}$, and $0.95 \lambda_{u} r^{2}$ if we consider only users meeting the criterion on the received signal strength.

\section{CONCLUSION}

We proposed a new point process that is more realistic than the Poisson Point Process to model an integrated FTC/WiFi network. The FTC/Wi-Fi network is used to extend the coverage of the macro base station to poorly covered (or 


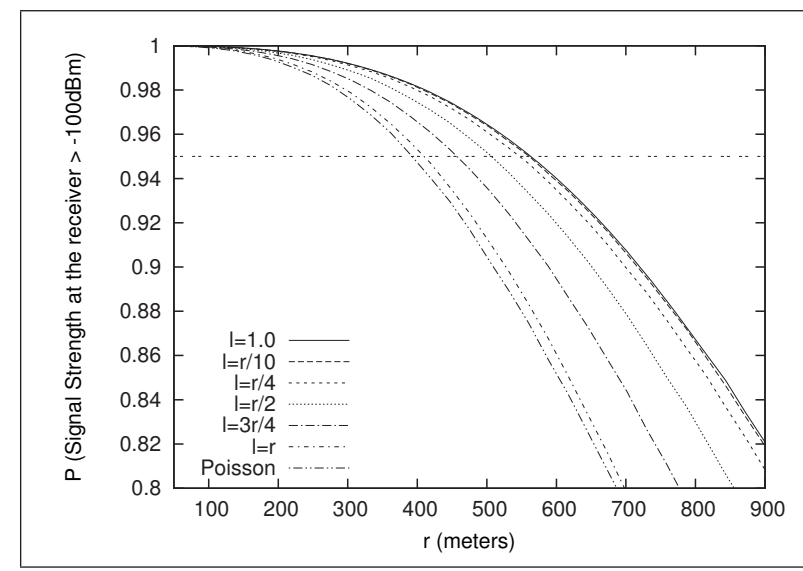

(a) $\mathbb{P}$ (Received signal strength $>-110 \mathrm{dBm}$ ) for a typical user

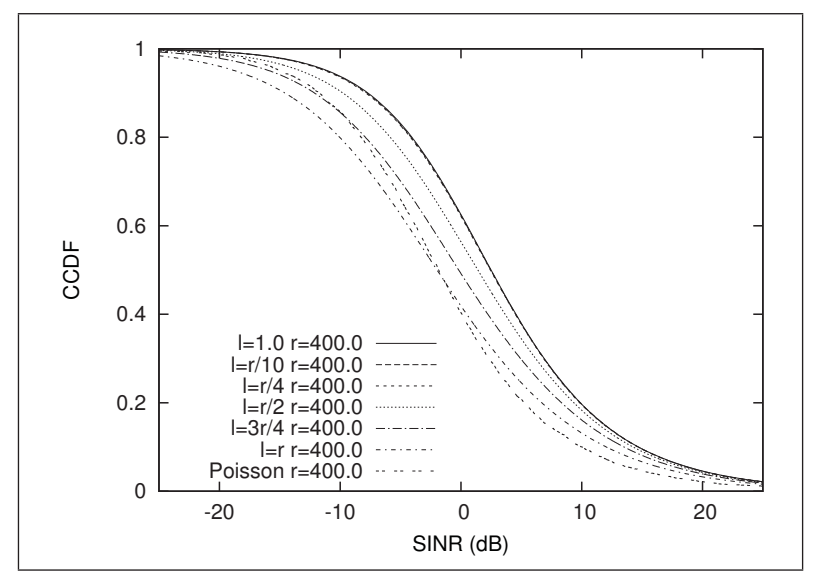

(b) CCDF of the SINR.

Fig. 4. CCDF of SINR and received signal strength

uncovered) areas. The so formed mesh Wi-Fi network is used as an access network for the users which are very far from the macro base station.

The obtained model has the benefit to offer a more regular coverage, and inhibit the property of hard-core point processes where points cannot be arbitrarily close to each other. Coverage and outage have been derived for this model, offering some insights on the deployment of such networks.

We have shown that this more realistic model exhibits a better coverage than a Poisson point process with same intensity both in terms of SINR and received signal strength. Our point process depends on two main parameters: $r$ which is related to the point intensity, and 1 that sets the minimal distance between the points. The parameter $r$ mainly impacts the received signal strength at a receiver whereas 1 impacts SINR distribution. It is thus possible to propose dimensioning rules of the femtocell networks according to these two criteria. For example, the results show that assuming a target signal strength of $-100 \mathrm{dBm}$ without considering fading, 95\% of the users will experience a signal strength higher than this threshold while maximum value of $r$ varies between 400 to 570 meters. Moreover, this work may help in the design of algorithms that switch-off certain nodes to save energy while ensuring the proper operation of the network in terms of connectivity and coverage. A direct extension of this study might involve combining the proposed point process within a model allowing us to evaluate precisely the gain in energy when the femtocells- Wi-Fi nodes are switched-off.

\section{REFERENCES}

[1] J. Andrews, F. Baccelli, and R. Ganti, "A tractable approach to coverage and rate in cellular networks," IEEE Transactions on Communications, vol. 59, no. 11, pp. 3122-3134, 2011.

[2] H.-S. Jo, Y. J. Sang, P. Xia, and J. G. Andrews, "Heterogeneous cellular networks with flexible cell association: A comprehensive downlink sinr analysis," CoRR, vol. abs/1107.3602, 2011.

[3] W. C. Cheung, T. Quek, and M. Kountouris, "Throughput optimization, spectrum allocation, and access control in two-tier femtocell networks,' IEEE Journal on Selected Areas in Communications, vol. 30, no. 3 , pp. 561-574, 2012.
[4] J. G. Andrews, H. Claussen, M. Dohler, S. Rangan, and M. C. Reed, "Femtocells: Past, present, and future," IEEE Journal on Selected Areas in Communications, vol. 30, no. 3, pp. 497-508, 2012.

[5] H. Wang, X. Zhou, and M. C. Reed, "Analytical evaluation of coverageoriented femtocell network deployment," CoRR, vol. abs/1305.3356, 2013.

[6] W. Wang, G. Yu, and A. Huang, "Cognitive radio enhanced interference coordination for femtocell networks," IEEE Communications Magazine, vol. 51, no. 6, 2013.

[7] M. Bennis, M. Simsek, A. Czylwik, W. Saad, S. Valentin, and M. Debbah, "When cellular meets wifi in wireless small cell networks," Communications Magazine, IEEE, vol. 51, no. 6, 2013.

[8] "Running for the coverage: A review of femtocells." http://www. arubanetworks.com/pdf/technology/whitepapers/WP_FEMTO.pdf, 2009.

[9] H. Dhillon, R. Ganti, and J. Andrews, "A tractable framework for coverage and outage in heterogeneous cellular networks," in Information Theory and Applications Workshop (ITA), 2011, pp. 1-6, 2011.

[10] A. Busson and G. Chelius, "Capacity and interference modeling of csma/ca networks using ssi point processes," Telecommunication Systems, pp. 1-15, 2013.

[11] D. Stoyan, S. Kendall, and J. Mecke, Stochastic geometry and its applications, second edition. John Wiley \& Sons, 1995. 